\title{
Application of cone-beam computed tomography (CВCT) for diagnosis and management of mandibular condyle fractures
}

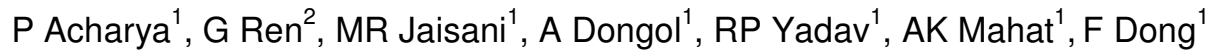 \\ Oral and Maxillofacial Surgery Department, BPKIHS ${ }^{1}$, Hebei Medical University ${ }^{2}$
}

\begin{abstract}
Background: Cone beam computed tomography (CBCT) provides precise imaging of temporomandibular joint anatomy without superimposition and distortion. CBCT is relatively a new imaging modality and used commonly in dental practice. Objective: The aim of this study is to present detailed imaging of temporomandibular joint in case of condyle fracture using CBCT for its use in diagnosis, surgical planning and evaluation of treatment. Method: In our study, we evaluated 3D - CBCT (three dimensional Cone beam computed tomography) examinations of 18 patients with mandibular condyle fractures. All of the fractures in our cases were overlooked on CBCT, thus providing axial, coronal and para-sagittal imaging of condylar head including 3D dimensional volumetric images of the condyle and surrounding structures. Result: Out of 18 condylar fracture patient's CBCT, 8 radiographs showed condylar head (intracapsular) fracture, 2 condylar neck, 8 subcondylar fracture. 13 condylar fractures were unilateral and 5 were bilateral fracture. 9 of the condylar fractures were not associated with the mandible fracture. Out of the 9 associated condylar fractures; 3 were associated with symphysis fracture, 4 associated with parasymphysis fracture, 1 associated with body fracture and remaining 1 associated with mandibular angle fracture. 6 of the condylar fracture showed no signs of displacement of the fractured part and among 12 displaced condylar fracture parts 8 medially and 4 laterally displaced recorded in CBCT. All of the patients were given treatment on the basis of CBCT diagnosis and the results obtained from it was clinical satisfactory without complaints. Conclusion: We concluded that CBCT is the latest sophisticated technology which provides clear image of condylar head without superimposition of other structures, presented supplementary information for a more effective diagnosis and management of mandibular condyle fractures.
\end{abstract}

Key words: Condylar fracture, cone beam computed tomography, mandible

\footnotetext{
Address for correspondence

Dr. Pradip Acharya

Department of Oral and Maxillofacial Surgery

B.P. Koirala Institute of Health Sciences, Dharan

Email: pradeepddh@yahoo.com
} 


\section{Introduction}

The temporomandibular joint (TMJ) has many complex anatomical and functional features. Therefore, caution should be exercised when diagnosing TMJ dysfunctions, such as condylar fractures after facial trauma. Mandibular fractures may be caused by direct or indirect mechanical force to the mandible in facial trauma. The frequency of condylar fractures is about $20-62 \%$ of all incidences of mandibular fractures and varies among epidemiology studies.

Fractures involving the mandibular articular process have been classified from the standpoint of diagnosis or practical purpose. MacLennan ${ }^{1}$ dichotomized fractures of the mandibular articular process into intracapsular and extracapsular fracture. Several studies have also classified these fractures into three types, namely condylar head, condylar neck, and subcondylar fractures. ${ }^{2-4}$ In addition, some classifications in accordance with the fracture lines or fragment dislocation have been proposed. ${ }^{1,5,6}$ Various radiographic methods have been used for diagnosing TMJ components. Panoramic tomography is widely used for the screening of orofacial trauma as well as other diseases. ${ }^{7}$ Computed tomography (CT), which provides the clearest definition of bone structure, is the best modality for detecting fracture lines. Cone-beam CT (CBCT) is also used for evaluating small bone and tooth structures. The benefits of CT and CBCT in oral and maxillofacial diagnosis have been demonstrated in clinical and experimental studies. $^{8,9,10}$ In clinical image diagnosis, the detectability of condylar fractures depends on the position of the fracture line, the degree of displacement of the fracture pieces, and the imaging methods. Panoramic tomography and plane radiography often overlook evidence of condylar fractures. Therefore, we conducted this study to provide some imaging features that clarify the indication for CBCT for a condylar fracture. The purposes of this report are to show the usefulness of CBCT in diagnosing condylar fractures and to describe the imaging features of condylar fractures to provide the guideline for treatment.

\section{Methods}

Patient: Patients with known fractures of the mandible treated at department of Oral and Maxillofacial Surgery, College of Stomatology, Hebei Medical University, NO. 383 East Zhongshang road, Shijiazhuang, China, from April 2011 to March 2013 were included in this study. A total of 49 patients with a variety of maxillofacial injuries based on history, clinical signs and symptoms of facial fracture and Cone Beam Computed Tomographical examination (CBCT) were included in this study. All the images were examined and recorded, 18 patients were diagnosed with condylar fracture, who ranges 
in age from 3 to 67 years old (average 20.6 years).The study population comprised 15 $(83.3 \%)$ males and $3(16.7 \%)$ females (figure13, table-1). Several parameters were considered such as the number of fractures, the age and sex of the patient, the affected side, (unilateral or bilateral), the level of the fracture (condylar head, condylar neck and subcondylar), the association with other fractures of the mandible, and treatment. This report, however, is taken from the patients that were admitted in the Inpatient and an outpatient department basis. Data source was the medical records of the patients included in the study. Reports given by radiologists were compared with the final diagnosis obtained from panoramic radiographs were sufficient for diagnosis in most cases, but we did CBCT in all patients to view all the coronal, sagittal and axial views as well as 3D panoramic and lateral view to ensure the accuracy of diagnosis.

Imaging methods: All subjects were seated during the exam and were oriented to have their heads positioned with the Frankfurt horizontal plane parallel to the floor. The CBCT scans were taken with the CBCT device (KAVO 3D Exam, Model 17-19, Imaging Sciences International, Hatfield, $\mathrm{Pa}$ USA) consists of a standard high frequency anode X-ray tube (120 kVp, 3-7mA) and $23.2 \times 17 \mathrm{~cm}$ amorphous silicone (a-Si) flat panel image detector, and uses a cone- shaped $x$-ray collimator with a 15 degree aperture centered on an x-ray area detector. It acquires a raw data with a single $360^{\circ}$ rotation in 17.8 seconds around the patient's head, with a projection at every $1^{\circ}$ step. The images are captured by an amorphous silicone flat panel image detector and stored on a hard drive. The x-ray emission time was 17.8 seconds. Exposures were made with 5.0 $\mathrm{mA}, 120 \mathrm{kVp}$ and an exposure time of 17.8 seconds. Major reconstruction time of $\leq 2$ minutes and real-time minor reconstruction time were applied. All the images were obtained with 576 projections. The voxel dimension in the reconstructed image was $0.3 \times 0.3 \times 0.3 \mathrm{~mm}$ and the reconstruction shape was cylindrical.

\section{Treatment}

Out of 18 patients with condylar fracture, 8 patients with non dislocated fracture high condylar intracapsular fracture, or fracture without functional impairment such as malocclusion and limitation of mouth opening and young patient with intracapsular fracture and minimal displaced are treated in outpatient department with intraoperative MMF (Maxillomandibular fixation) for 4 weeks.

Remaining 10 patients with malocculsion and additional mandibular fracture were treated by open reduction and internal fixation with in in-patient department; none of the patients 
were treated with pre-aurical approach. Postramal approach was done in 4 patients for internal fixation of subcondylar fracture and intraoral approach was done in 6 patients for internal fixation of mandible fracture associated with condylar fracture. In 10 patients arch bar used for intraoperative MMF (Maxillomandibular fixation) for 2 weeks.

The modified protocol treatment involving intra-articular injection of HA (Hyaluronic Acid) was given in all the 18 patients who were with condylar fracture. The first injection of HA was given on the first visit, followed by 1 injection weekly for next 2 weeks.

\section{Results}

Total 18 patients were diagnosed as condylar fracture. Sex wise dixtribution is given in table 1.

Table 1: Sex wise determination of patients with condylar fracture

\begin{tabular}{|l|c|c|}
\hline Gender & No. of patents & Percentage \\
\hline Male & 15 & 83.3 \\
\hline Female & 3 & 16.7 \\
\hline Total & 18 & 100 \\
\hline
\end{tabular}
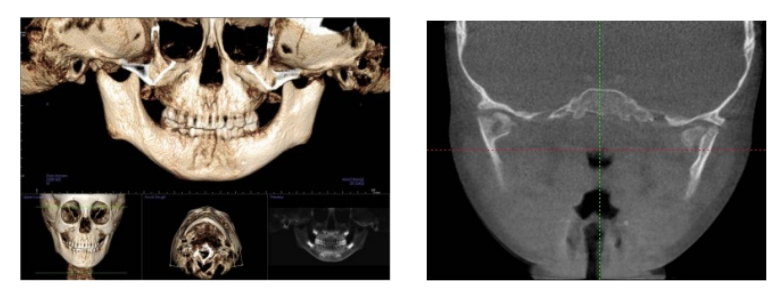

Fig-1a:3D volumetric image of 4 years old male patient shows the intracapsular condylar fracture associated with parasymphysis fracture line, here the condylar fracture is not visible.

Figure -1b: Coronal view shows both intracapsular condylar fracture where as right side is not dislocated and left side is minimally dislocated medially.
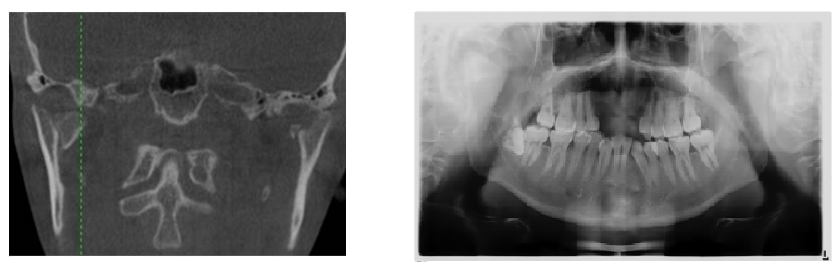

Figure -2a: Coronal views of CT scan shows the bilateral intracapsular condylar fracture with medially displaced.

Figure -2b: OPG of the patient where condylar fracture cannot be detected clearly due to the superimposition of images.

Out of 18 condylar fracture patient's CBCT, 8 (44.5\%) radiographs showed condylar head (intracapsular) fracture (figure-1a, 1b, 2a, table-2), 2 (11\%) condylar neck, 8 (44.5\%) subcondylar fracture (figure-3, table-2).

Table 2: Anatomical wise determination of condylar fracture

\begin{tabular}{|l|l|l|}
\hline Location & No. of & Percentage \\
\hline
\end{tabular}




\begin{tabular}{|l|l|l|}
\hline & patients & \\
\hline Intracapsular & 8 & 44.5 \\
\hline Condylar neck & 2 & 11 \\
\hline Subcondylar & 8 & 44.5 \\
\hline Total & 18 & 100 \\
\hline
\end{tabular}

$13(72.2 \%)$ condylar fracture were unilateral and $5(27.8 \%)$ were bilateral fracture (table3). $9(50 \%)$ of the condylar fractures were not associated with the mandible fracture (figure4).

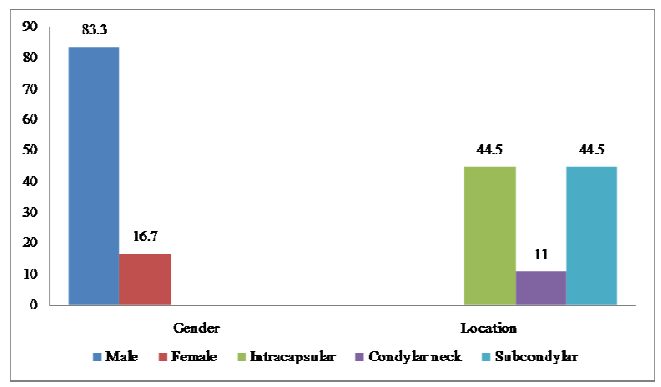

Figure-3: Bar chart showing the gender and location of condylar fracture in percentage.

Table 3: Condylar fracture involvement of site

\begin{tabular}{|l|l|l|}
\hline Site & No. of patients & Percentage \\
\hline Unilateral & 13 & 72.2 \\
\hline Bilateral & 5 & 27.8 \\
\hline Total & 18 & 100 \\
\hline
\end{tabular}

Out of 18 condylar fracture patient's CBCT, 6 (33.3\%) of the condylar fracture showed no signs of displacement of the fractured part and among 12 displaced condylar fracture parts 8 (44.5\%) medially and 4 (22.2\%) laterally displaced recorded in CBCT (table4).

Table 4: Condylar fracture associated with mandible fracture

\begin{tabular}{|l|c|c|}
\hline \multicolumn{1}{|c|}{ Associated } & $\begin{array}{c}\text { No. of } \\
\text { patients }\end{array}$ & Percentage \\
\hline Not associated & 9 & 50 \\
\hline Symphysis & 3 & 16.7 \\
\hline Parasymphysis & 4 & 22.3 \\
\hline Body of mandible & 1 & 5.5 \\
\hline Angle of mandible & 1 & 5.5 \\
\hline Total & 18 & 100 \\
\hline
\end{tabular}

Out of the 9 associated condylar fractures; 3 (16.7\%) were associated with symphysis fracture, 4 (22.3\%) associated with parasymphysis fracture, 1 (5.5\%) associated with body fracture and remaining 1 (5.5\%) associated with mandibular angle fracture (figure-4, table-5).

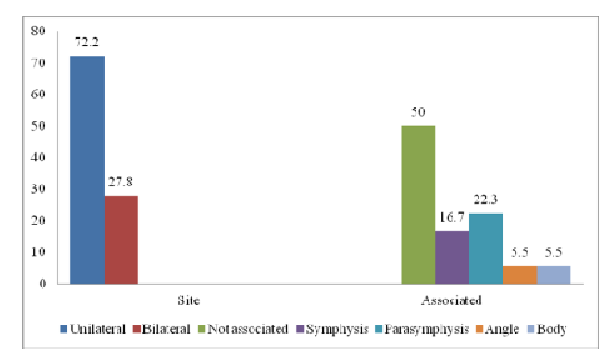

Figure-4: Bar chart showing the site and associated condylar fracture with other parts of mandible in percentage 
Table 5: Location of fracture part

\begin{tabular}{|l|c|c|}
\hline $\begin{array}{c}\text { Displaced of } \\
\text { fracture parts }\end{array}$ & $\begin{array}{c}\text { No. of } \\
\text { patients }\end{array}$ & Percentage \\
\hline No displaced & 6 & 33.3 \\
\hline Medial displaced & 8 & 44.5 \\
\hline Lateral displaced & 4 & 22.2 \\
\hline Total & 18 & 100 \\
\hline
\end{tabular}

At 23 months of after surgery, TMJ function was not impaired in any patient, all patients with a mouth opening of more than $38 \mathrm{~mm}$, none of the patients has pain in TMJ on rest, 3 patients only complained of mild pain on fracture side during maximum opening of mouth in the TMJ region. On examination at 23 months of after surgery, Occlusion was normal, no deviation of mandible found, masticatory functions was normal, no retardation of mandible growth in children, no facial nerve injuries, ramal height was normal. All 18 patients who had received HA injection showed no symptoms of TMJ adhesion and have normal joint movement. The posttreatment $\mathrm{CBCT}$ revealed that the condylar fracture had well healed. Infiltration of HA into the TMJ proved beneficial to the patients with condylar fractures for better masticatory function.

\section{Discussion}

Radiologic examination is essential for evaluating the mandibular condylar fracture.
Conventional radiograph such as OPG, lateral skull view, posterior anterior views, conventional CT and CBCT are useful in the initial evaluation of suspected condylar fractures.

OPG is commonly used in emergency patient, where the advance radiographs are not available. OPG allow examination of the entire mandible, both TMJ, the maxilla, and the maxillary sinuses. Though panoramic radiograph has been using widely, it has many shortcomings related to the reliability and accuracy of size, location, and form of the images created. OPG provide the essential preliminary information, but these will not be sufficient for critical evaluation of the condylar fracture itself because of the superimpositions of surrounding structures on the roentgenogram (figure-2b). The panoramic radiography creates only flat, two dimensional, supero-inferior or posteroanterior images and it suffers from superimposition of all structures that lie in the path between the X-ray source and the film and the detector. It compresses a three dimensional anatomy into a two dimensional image. Therefore, it results in misdiagnosis of an imaginary condylar fracture.

When OPG do not show clearly the degree of displacement, type of fracture or degree of comminution, for example, in suspected fractures of the condylar head and neck, CT or conebeam CT is indicated. ${ }^{11}$ CBCT has 
recently been developed as an alternative to conventional CT for dental and maxillofacial diagnostic osseous tasks. CBCT may prove to be more efficient and economical than either conventional tomography or CT for oral diagnostics and is a modern imaging technique that has the advantage of being associated with a low level of metal artifacts. $^{12}$ Using a cone-shaped x-ray beam rather than the linear fan beam of conventional CT, a CBCT scanner takes just one revolution around the patient to capture these multi planner views. CBCT can take both jaws in 3.6-6 seconds of actual exposure time. This represents significantly less radiation than one would receive with a full series of digital periapical radiographs, and is relatively equivalent to bite wing radiographs. The dose of radiation needed for a CBCT is much lower than for a conventional CT. ${ }^{13}$ CBCT allows anatomic entities to be viewed in three dimensions and be included or excluded in the final image by simple digital manipulation(figure-1a). ${ }^{14}$ CBCT is particularly useful for evaluation of the temporo-mandibular joints. ${ }^{15,16}$ The joints may be visualized in volumetric views as well as sectional views. We applied CBCT for followup assessment in almost all patients with condylar fractures. ${ }^{17}$.

The diagnostic specificity of CBCT for the evaluation of the TMJ has been studied and it seems to be a significant improvement over conventional means. CBCT offered a diagnostic specificity of $0.95 \pm 0.05$ compared with conventional panoramic radiography (0.64 \pm 0.11$)$, TMJ-specific panoramic radiography $(0.55 \pm 0.11)$ and tomograms (0.58 \pm 0.15). Schimming et al. (18)determined that the accuracy of conventional radiography with both panoramic tomography and posteroanterior mandibular radiography was $100 \%$ for detecting condylar fractures, although identification of the fragment position and dislocation was superior in CBCT compared with conventional radiography

\section{Conclusion}

All of the fractures in our cases were overlooked on CBCT, which shows clear images of condylar head without superimposition of other structure, thus providing axial, coronal and para-sagittal imaging of condylar head including 3D dimensional volumetric images of condyle and surrounding structure. In our study sample, the treatment of the condylar fracture was given based on the diagnosis made from CBCT scans. Post-treatment results of the patients were clinical satisfactory.

CBCT is suitable for detecting fracture lines and minimal dislocation of fragments, which provide fine details for more accurate diagnosis, treatment planning and more predictable treatment outcomes as well as 
performing follow-up examinations. CBCT is a sophisticated technology, which in its third generation, sets the gold standard again in comparision to conventional radiograph in which volumetric imaging system provides dentists and specialists complete views of TMJ.

\section{References}

1. MacLennan WD. Fractures of the mandibular condylar process. Br J Oral Surg. 1969;7(1):31-9.

2. Lindahl L. Condylar fractures of the mandible. I. Classification and relation to age, occlusion, and concomitant injuries of teeth and teeth-supporting structures, and fractures of the mandibular body. Int $\mathrm{J}$ Oral Surg. 1977;6(1):12-21.

3. Silvennoinen $U$, lizuka $T$, Lindqvist $C$, Oikarinen K. Different patterns of condylar fractures: an analysis of 382 patients in a 3-year period. J Oral Maxillofac Surg. 1992;50(10):1032-7.

4. Newman L. A clinical evaluation of the long-term outcome of patients treated for bilateral fracture of the mandibular condyles. $\mathrm{Br} \mathrm{J}$ Oral Maxillofac Surg. 1998;36(3):176-9.

5. Loukota RA, Eckelt U, De Bont L, Rasse M. Subclassification of fractures of the condylar process of the mandible. $\mathrm{Br} \mathrm{J}$ Oral Maxillofac Surg. 2005;43(1):72-3.

6. Hyde N, Manisali M, Aghabeigi B, Sneddon K, Newman L. The role of open reduction and internal fixation in unilateral fractures of the mandibular condyle: a prospective study. $\mathrm{Br} \mathrm{J}$ Oral Maxillofac Surg. 2002;40(1):19-22.

7. Boeddinghaus $R$, Whyte $A$. Current concepts in maxillofacial imaging. Eur J Radiol. 2008;66(3):396-418.

8. Barghan S, Tetradis S, Mallya S. Application of cone beam computed tomography for assessment of the temporomandibular joints. Aust Dent J. 2012;57(1):109-18.

9. Zain-Alabdeen EH, Alsadhan RI. A comparative study of accuracy of detection of surface osseous changes in the temporomandibular joint using multidetector CT and cone beam CT. Dentomaxillofac Radiol. 2012;41(3):185-91.

10. $\mathrm{Hu} \mathrm{H}, \mathrm{He} H D$, Foley WD, Fox SH. Four multidetector-row helical CT: image quality and volume coverage speed. Radiology. 2000;215(1):55-62.

11. De Vos W, Casselman J, Swennen GRJ. Cone-beam computerized tomography (CBCT) imaging of the oral and maxillofacial region: a systematic review of the literature. Int $\mathrm{J}$ 
Oral Maxillofac Surg. 2009;38(6):60925.

12. Stuehmer C, Essig $\mathrm{H}$, Bormann $\mathrm{K}-\mathrm{H}$, Majdani O, Gellrich N-C, Rücker M. Cone beam CT imaging of airgun injuries to the craniomaxillofacial region. Int $\mathrm{J}$ Oral Maxillofac Surg. 2008;37(10):903-6.

13. Kaban LB, Mulliken JB, Murray JE. Facial fractures in children: an analysis of 122 fractures in 109 patients. Plast Reconstr Surg. 1977;59(1):15-20.

14. Guttenberg SA. Oral and maxillofacial pathology in three dimensions. Dent Clin North Am. 2008;52(4):843-73, viii.

15. Honda K, Arai $\mathrm{Y}$, Kashima M, Takano Y, Sawada K, Ejima K, et al. Evaluation of the usefulness of the limited cone-beam CT (3DX) in the assessment of the thickness of the roof of the glenoid fossa of the temporomandibular joint.
Dentomaxillofac

Radiol. 2004;33(6):391-5.

16. Nakajima A, Sameshima GT, Arai $Y$, Homme Y, Shimizu N, Dougherty $\mathrm{H}$. Two- and three-dimensional orthodontic imaging using limited cone beam-computed tomography. Angle Orthod. 2005;75(6):895-903.

17. Honey OB, Scarfe WC, Hilgers MJ, Klueber K, Silveira AM, Haskell BS, et al. Accuracy of cone-beam computed tomography imaging of the temporomandibular joint: comparisons with panoramic radiology and linear tomography. Am J Orthod Dentofacial Orthop. 2007;132(4):429-38.

18. Schimming R, Eckelt $U$, Kittner $T$. The value of coronal computer tomograms in fractures of the mandibular condylar process. Oral Surg Oral Med Oral Pathol Oral Radiol Endod. 1999;87(5):632-9. 